\title{
Smooth muscle tumors of the gastrointestinal tract: an analysis of prognostic features in 407 cases
}

\author{
Lindsay Alpert ${ }^{1} \cdot$ Ram Al-Sabti ${ }^{1} \cdot$ Rondell P. Graham $\mathbb{B}^{2} \cdot$ Rish K. Pai $\mathbb{1}^{3} \cdot$ Raul S. Gonzalez $\mathbb{D}^{4} \cdot$ Xuefeng Zhang $^{5}$. \\ Vanessa Smith $\mathbb{1}^{5} \cdot$ Hanlin L. Wang ${ }^{6} \cdot$ Lindsey Westbrook $\mathbb{1}^{6} \cdot$ John R. Goldblum ${ }^{7} \cdot$ Ahmed Bakhshwin $^{7}$. \\ Sindhu Shetty ${ }^{7} \cdot$ David S. Klimstra $^{8} \cdot$ Jinru Shia $\mathbb{1}^{8} \cdot$ Gokce Askan $^{8} \cdot$ Marie E. Robert $^{9} \cdot$ Courtney Thomas $^{9}$. \\ Wendy L. Frankel ${ }^{10} \cdot$ Mohammed Alsomali $^{10} \cdot$ Catherine Hagen $^{11} \cdot$ Mohamed E. Mostafa ${ }^{11} \cdot$ Michael M. Feely $^{12}$. \\ Naziheh Assarzadegan ${ }^{12} \cdot$ Joseph Misdraji ${ }^{13}$. Angela R. Shih ${ }^{13}$. Diana Agostini-Vulaj $\mathbb{D}^{14}$ - Jeanne M. Meis ${ }^{15}$. \\ Sherry Tang ${ }^{15}$. Deyali Chatterjee ${ }^{16}$. Liang-I Kang ${ }^{16}$. John Hart ${ }^{1}$ - Sang Mee Lee ${ }^{1}$. Theresa Smith ${ }^{17}$.

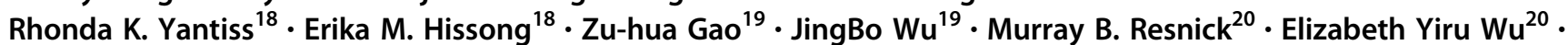 \\ Reet K. Pai ${ }^{21}$ - Lei Zhao ${ }^{22}$ • Leona A. Doyle ${ }^{22}$. Shefali Chopra ${ }^{23}$ - Nicole C. Panarelli ${ }^{24}$. Shaomin $\mathrm{Hu}^{24}$. \\ Teri A. Longacre ${ }^{25}$. Shyam Sampath Raghavan $\mathbb{1}^{25}$. Gregory Y. Lauwers ${ }^{26}$ - Masoumeh Ghayouri ${ }^{26}$. \\ Harry S. Cooper ${ }^{27} \cdot$ Rajeswari Nagarathinam ${ }^{27}$ - Andrew M. Bellizzi' ${ }^{28} \cdot$ Sanjay Kakar $^{29} \cdot$ Mojgan Hosseini $^{30}$. \\ Juan Rong ${ }^{30} \cdot$ Joel K. Greenson ${ }^{31} \cdot$ Laura W. Lamps $^{31} \cdot$ Zachary Dong $^{32} \cdot$ Mary P. Bronner $^{32}$
}

Received: 7 November 2019 / Revised: 15 January 2020 / Accepted: 27 January 2020 / Published online: 12 February 2020

(c) The Author(s), under exclusive licence to United States \& Canadian Academy of Pathology 2020

\begin{abstract}
Smooth muscle tumors represent the second most common mural mesenchymal neoplasm in the gastrointestinal tract, but established criteria for prognostic assessment of these tumors are lacking. A large cohort of surgically resected intramural gastrointestinal smooth muscle tumors from 31 institutions was analyzed to identify potential prognostic features. Pathologic features were assessed by expert gastrointestinal and/or soft tissue pathologists at each center. Immunohistochemical confirmation was required. A total of 407 cases from the esophagus $(n=97,24 \%)$, stomach $(n=180,44 \%)$, small bowel $(n=74,18 \%)$, and colorectum $(n=56,14 \%)$ were identified. Patients ranged in age from 19 to 92 years (mean 55 years), with a slight female predominance (57\%). Mean tumor size was $5.4 \mathrm{~cm}$, with the largest tumor measuring $29 \mathrm{~cm}$. Disease progression following surgery, defined as local recurrence, metastasis, or disease-related death, occurred in 56 patients (14\%). Colorectal tumors were most likely to progress, followed by small bowel and gastric tumors. None of the esophageal tumors in this series progressed. Receiver operator characteristic analysis identified optimal cutoffs of $9.8 \mathrm{~cm}$ and 3 mitoses $/ 5 \mathrm{~mm}^{2}$ for discriminating between progressive and non-progressive tumors. Histologic features strongly associated with progression by univariate analysis included moderate-to-severe atypia, high cellularity, abnormal differentiation (defined as differentiation not closely resembling that of normal smooth muscle), tumor necrosis, mucosal ulceration, lamina propria involvement, and serosal involvement $(P<0.0001$ for all features). Age, sex, and margin status were not significantly associated with progression $(P=0.23,0.82$, and 0.07 , respectively). A risk assessment table was created based on tumor site, size, and mitotic count, and Kaplan-Meier plots of progressionfree survival for each subgroup revealed progression-based tiers. Based on our findings, it appears that nonesophageal gastrointestinal smooth muscle tumors measuring $>10 \mathrm{~cm}$ and/or showing $\geq 3$ mitoses $/ 5 \mathrm{~mm}^{2}$ may behave aggressively, and therefore close clinical follow-up is recommended in these cases.
\end{abstract}

\section{Introduction}

Lindsay Alpert

lindsay.alpert@uchospitals.edu

Extended author information available on the last page of the article.
For the past two decades, gastrointestinal stromal tumors (GISTs) have dominated the field of gastrointestinal (GI) tract mesenchymal neoplasms. As a result of Miettinen and Lasota's foundational, large-scale studies of these tumors at various sites within the GI tract, a risk assessment tool is 
widely available to help predict the behavior of GISTs based on their location, size, and mitotic rate [1-4]. However, the situation is much less straightforward when pathologists encounter the second most common mural mesenchymal tumor of the GI tract: the true smooth muscle tumor.

Historically, published studies on intramural GI smooth muscle tumors have primarily consisted of small case series [5-13]. Although the existing studies often separate these lesions into benign and malignant tumors, the criteria by which these classifications have been made are not well defined. In addition, some studies have described a group of GI tract smooth muscle tumors that behave in a malignant fashion despite low mitotic rates, highlighting the need for additional analysis of potential prognostic features in a larger series of cases $[8,10]$.

We initiated a multi-institutional study of intramural smooth muscle tumors of the GI tract in an attempt to elucidate the issues raised by prior case series. By analyzing a large cohort of documented true smooth muscle tumors with long-term follow-up, we aimed to better understand their natural history, and to correlate their behavior with clinicopathologic features in order to create a risk assessment tool similar to that used for GISTs.

\section{Materials and methods}

\section{Case selection}

Smooth muscle tumors resected from the esophagus, stomach, small intestine, and colorectum were identified from 31 institutions in North America, each of which obtained institutional review board approval. Immunohistochemical confirmation of smooth muscle differentiation was required, including desmin and/or smooth muscle actin immunoreactivity. KIT (CD117) and/or DOG1 staining to exclude GIST was also performed in the majority of cases and was required in cases with ambiguous lineage. Negative Epstein-Barr virus (EBV) staining was required in cases from solid organ transplant patients and patients with acquired immunodeficiency syndrome (AIDS) in order to exclude EBV-associated smooth muscle tumors. Tumors measuring less than $0.5 \mathrm{~cm}$ in greatest dimension and muscularis mucosae leiomyomas were excluded from the study, as were excisional biopsies, polypectomies, and endoscopic resections. Anal tumors and tumors that appeared to arise from outside of the GI tract (mesenteric or serosal tumors, metastatic tumors, and parasitic leiomyomas) were also excluded. Cases from female patients with a clinical history suspicious for involvement by a smooth muscle tumor of gynecologic tract origin, including cases with positive estrogen receptor staining, were excluded as well.

\section{Case analysis}

Hematoxylin and eosin-stained slides (range 1-25, median 4) for each case were evaluated by experienced GI and/or soft tissue pathologists at each institution. Tumors located at the esophagogastric junction were classified as esophageal tumors. If more than one smooth muscle tumor was present in a given resection, the largest tumor was used for analysis. Histologic features assessed included degree of cytologic atypia, cellularity, differentiation, mitotic activity, tumor necrosis, mucosal ulceration, layers of wall involved, and margin status.

Nuclear atypia was graded as none, mild, moderate, or severe based on the degree of nuclear enlargement, hyperchromasia, and/or pleomorphism, and its presence was also classified as focal $(<50 \%)$ or diffuse $(\geq 50 \%)$ (Fig. 1). Tumor cellularity was defined as low if it appeared equal to or lower than normal muscularis propria cellularity and high if it appeared higher than normal muscularis propria cellularity. Tumor differentiation was assessed per the approach outlined by the French Federation of Cancer Centers for the assessment of sarcomas [14]; specifically, tumors that closely resembled normal smooth muscle tissue were classified as having normal differentiation, tumors in which smooth muscle differentiation could be histologically identified even though they did not closely resemble normal smooth muscle tissue were classified as having abnormal differentiation, and tumors that could not be confidently recognized as having smooth muscle differentiation based on histology alone were classified as undifferentiated. Mitoses were counted in the most mitotically active $5 \mathrm{~mm}^{2}$ area. The presence of coagulative tumor necrosis was classified as $0 \%,<50 \%$, or $\geq 50 \%$. Lamina propria involvement was defined as tumor cells infiltrating between native mucosal structures (Fig. 2), and serosal involvement was defined as tumor penetrating the visceral peritoneum. Electronic images of representative features were provided to standardize the assessment, and there was also a robust online discussion with periodic electronic updates to help maintain consistency throughout the study.

\section{Outcome data}

Clinical data were retrieved from the electronic medical records of each institution. Tumor registries were also used to obtain follow-up data in some cases. Disease progression following surgery was defined as local recurrence, development of metastatic disease, or disease-related death. A follow-up period of at least 12 months was required for inclusion in the analysis, unless progression of disease was 
Fig. 1 Histologic spectrum of gastrointestinal smooth muscle tumor. A tumor with low cellularity, mild atypia, and differentiation closely resembling that of normal smooth muscle (a) compared with a tumor with high cellularity (b), a tumor with severe atypia (c), and an undifferentiated tumor $(\mathbf{d})$.
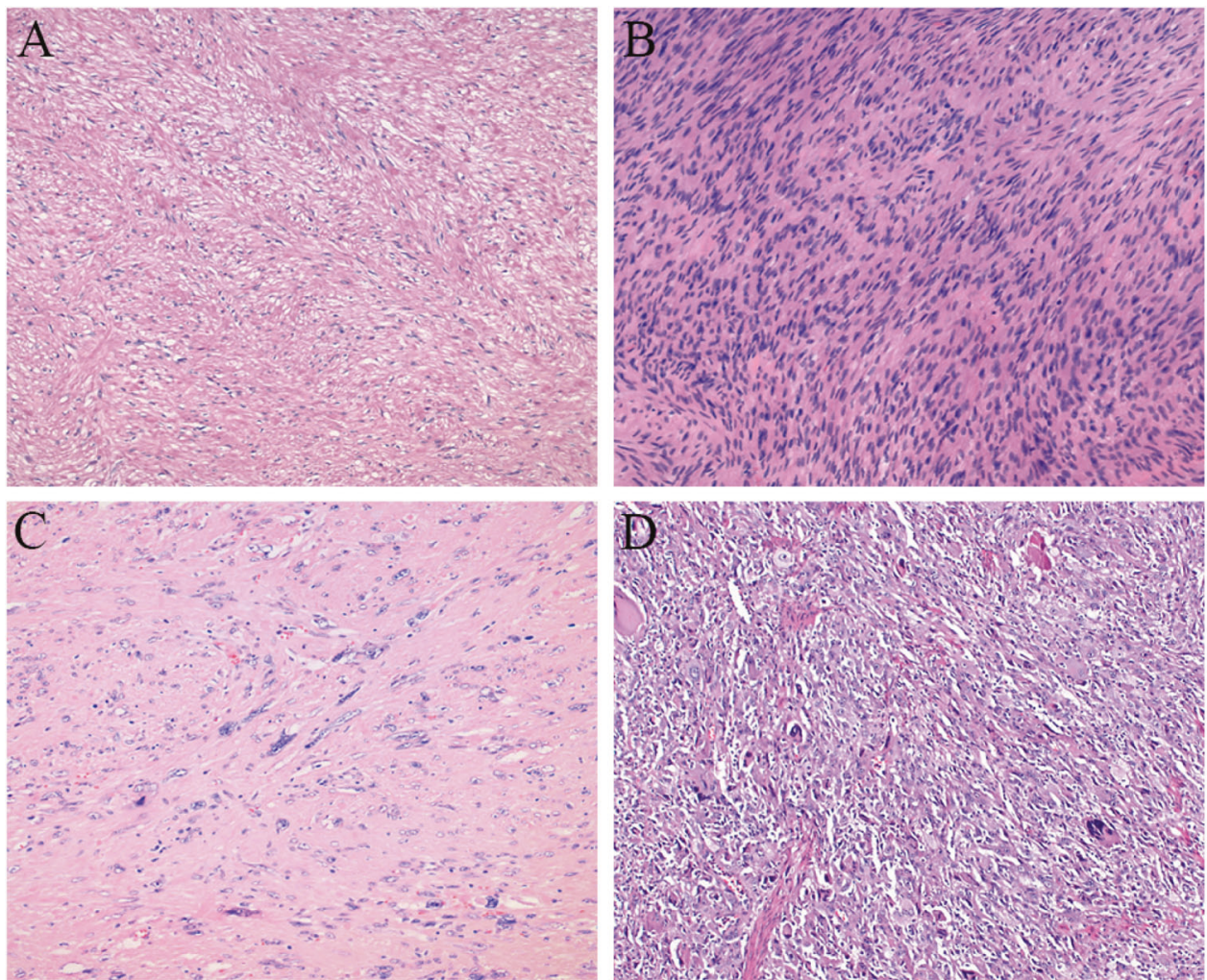

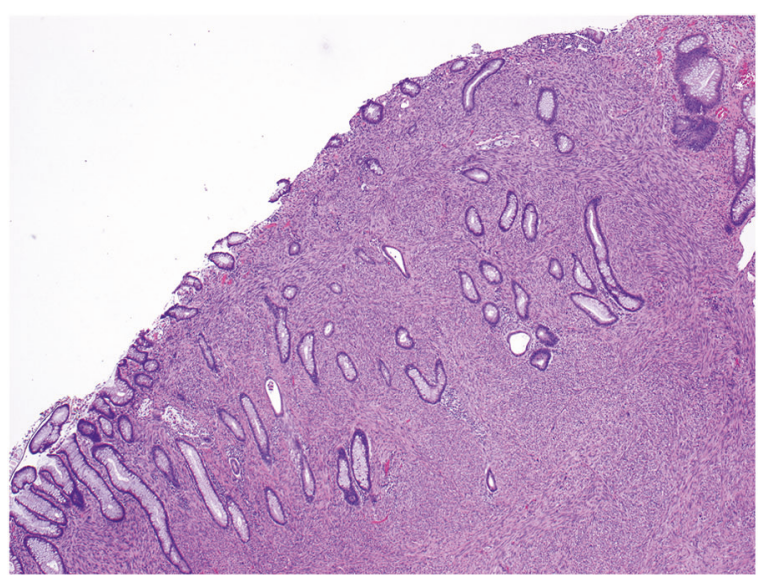

Fig. 2 Smooth muscle tumor demonstrating lamina propria invasion, with tumor cells infiltrating between colonic crypts.

noted earlier than 12 months. Patients for whom the cause of death could not be determined were not included in the analysis.

\section{Statistical analysis}

The association between pathologic features and disease progression was examined using Chi-square or Fisher's exact tests for categorical variables and $t$-tests for continuous variables. The receiver operating characteristic (ROC) method was used to determine the optimal cutoff point for continuous variables, including tumor size and mitotic activity. Based on these cutoffs, cases were divided into subgroups, and progression-free survival was estimated for each subgroup by the Kaplan-Meier method. All statistical analysis was performed using STATA version 15.1. A twosided $P<0.05$ was considered statistically significant.

\section{Results}

\section{Clinical features}

A total of 407 cases with sufficient data were identified and included in the analysis, of which 97 (24\%) were esophageal, $180(44 \%)$ were gastric, $74(18 \%)$ were small intestinal, and $56(14 \%)$ were colorectal (Table 1). There was a slight female predominance, with 231 females (57\%) and 176 males $(43 \%)$, resulting in a female-to-male ratio of 1.3:1; this slight female predominance was seen across all tumor sites. Patients ranged in age from 19 to 92 years, with a mean age of 55 years and a median age of 56 years. Only 22 patients were under age 30 at presentation, while 46 patients were over age 70 .

Clinical presentation data were available for 395 cases. The most common presentation was an incidentally detected mass lesion found during a medical procedure or clinical workup for another condition, which was the presentation in 115/395 cases $(29 \%)$. Of the patients with symptoms at presentation, 
Table 1 Association of clinicopathologic features with disease progression.

\begin{tabular}{|c|c|c|c|c|}
\hline & $\begin{array}{l}\text { All patients } \\
n=407\end{array}$ & $\begin{array}{l}\text { Progression } \\
n=56\end{array}$ & $\begin{array}{l}\text { No progression } \\
n=351\end{array}$ & $P$ value \\
\hline $\begin{array}{l}\text { Age, years, } \\
\text { mean } \pm S D\end{array}$ & $\begin{array}{l}55.4 \pm 14.4 \\
(19-92)\end{array}$ & $\begin{array}{l}57.7 \pm 15.7 \\
(21-86)\end{array}$ & $\begin{array}{l}55.1 \pm 14.2 \\
(19-92)\end{array}$ & 0.23 \\
\hline \multicolumn{5}{|l|}{ Sex } \\
\hline $\begin{array}{l}\text { Female } \\
\text { Male }\end{array}$ & $\begin{array}{l}57 \%(231) \\
43 \%(176)\end{array}$ & $\begin{array}{l}55 \%(31) \\
45 \%(25)\end{array}$ & $\begin{array}{l}57 \%(200) \\
43 \%(151)\end{array}$ & 0.82 \\
\hline $\begin{array}{l}\text { Tumor size, } \mathrm{cm} \\
\text { Mean } \pm \mathrm{SD}\end{array}$ & $\begin{array}{l}n=402 \\
5.4 \pm 4.8 \\
(0.5-29)\end{array}$ & $\begin{array}{l}n=54 \\
11.4 \pm 6.4 \\
(2.2-29)\end{array}$ & $\begin{array}{l}n=348 \\
4.4 \pm 3.7 \\
(0.5-29)\end{array}$ & $<0.0001$ \\
\hline \multicolumn{5}{|l|}{ Tumor site } \\
\hline $\begin{array}{l}\text { Esophagus } \\
\text { Stomach } \\
\text { Small bowel } \\
\text { Colon/rectum }\end{array}$ & $\begin{array}{l}24 \%(97) \\
44 \%(180) \\
18 \%(74) \\
14 \%(56)\end{array}$ & $\begin{array}{l}0 \%(0) \\
25 \%(14) \\
32 \%(18) \\
43 \%(24)\end{array}$ & $\begin{array}{l}28 \%(97) \\
47 \%(166) \\
16 \%(56) \\
9 \%(32)\end{array}$ & $<0.0001$ \\
\hline \multicolumn{5}{|l|}{ Atypia } \\
\hline $\begin{array}{l}\text { None/mild } \\
\text { Moderate/severe }\end{array}$ & $\begin{array}{l}69 \%(281) \\
31 \%(126)\end{array}$ & $\begin{array}{l}9 \%(5) \\
91 \%(51)\end{array}$ & $\begin{array}{l}79 \%(276) \\
21 \%(75)\end{array}$ & $<0.0001$ \\
\hline \multicolumn{5}{|l|}{ Cellularity } \\
\hline $\begin{array}{l}\text { Low } \\
\text { High }\end{array}$ & $\begin{array}{l}65 \%(264) \\
35 \%(143)\end{array}$ & $\begin{array}{l}4 \%(2) \\
96 \%(54)\end{array}$ & $\begin{array}{l}75 \%(262) \\
25 \%(89)\end{array}$ & $<0.0001$ \\
\hline \multicolumn{5}{|l|}{ Differentiation } \\
\hline $\begin{array}{l}\text { Normal } \\
\text { Abnormal } \\
\text { Undifferentiated }\end{array}$ & $\begin{array}{l}71 \%(287) \\
19 \%(78) \\
10 \%(42)\end{array}$ & $\begin{array}{l}9 \%(5) \\
43 \%(24) \\
48 \%(27)\end{array}$ & $\begin{array}{l}80 \%(282) \\
15 \%(54) \\
4 \%(15)\end{array}$ & $<0.0001$ \\
\hline \multicolumn{5}{|l|}{ Mitoses $/ 5 \mathrm{~mm}^{2}$} \\
\hline Median, mean (range) & $0,13.9(0-310)$ & $31.5,53(1-310)$ & $0,7.7(0-250)$ & $<0.0001$ \\
\hline \multicolumn{5}{|l|}{ Necrosis } \\
\hline $\begin{array}{l}\text { Absent } \\
\text { Present }\end{array}$ & $\begin{array}{l}76 \%(309) \\
24 \%(98)\end{array}$ & $\begin{array}{l}29 \%(16) \\
71 \%(40)\end{array}$ & $\begin{array}{l}83 \%(293) \\
17 \%(58)\end{array}$ & $<0.0001$ \\
\hline \multicolumn{5}{|l|}{ Ulceration } \\
\hline $\begin{array}{l}\text { Absent } \\
\text { Present }\end{array}$ & $\begin{array}{l}83 \%(324 / 392) \\
17 \%(68 / 392)\end{array}$ & $\begin{array}{l}63 \%(32 / 51) \\
37 \%(19 / 51)\end{array}$ & $\begin{array}{l}86 \%(292 / 341) \\
14 \%(49 / 341)\end{array}$ & $<0.0001$ \\
\hline Lamina propria involved & $14 \%(53 / 392)$ & $40 \%(21 / 52)$ & $9 \%(32 / 340)$ & $<0.0001$ \\
\hline Serosa involved & $11 \%(45 / 397)$ & $45 \%(23 / 51)$ & $6 \%(22 / 346)$ & $<0.0001$ \\
\hline Positive margin & $12 \%(41 / 335)$ & $20 \%(9 / 44)$ & $11 \%(32 / 295)$ & 0.07 \\
\hline
\end{tabular}

abdominal pain was most common $(93 / 395,24 \%)$, followed by dysphagia/dyspepsia/reflux $(67 / 395,17 \%)$ and GI bleeding (42/395, 11\%). Rarer presenting symptoms included chest pain, diarrhea, nausea, weight loss, and bowel obstruction, and many patients reported multiple symptoms at presentation. Not surprisingly, 45 of the 50 patients with a tumor measuring more than $10 \mathrm{~cm}$, for whom clinical presentation information was available, were symptomatic at presentation; abdominal pain was the most common presenting symptom in this group of patients with large tumors as well $(21 / 50,42 \%)$. Distant metastases were evident at presentation in only 13 cases, with liver being the most common site of metastatic disease at presentation (11/13).
Eight patients had multiple smooth muscle tumors identified at the time of resection. Three patients had two colonic smooth muscle tumors, three patients had multiple gastric tumors (2, 3, and "multiple"), and two patients had multiple esophageal tumors (2 and "multifocal"). None of the patients with multiple tumors had a known tumor syndrome, but genetic tumor-predisposing conditions were known to be present in nine other patients. Three patients had multiple endocrine neoplasia type 1 (MEN1), three patients had Lynch syndrome, one patient had familial adenomatous polyposis, one patient had hereditary diffuse gastric cancer, and one patient had a germline BRCA2 mutation. 


\section{Tumor size}

For the 402 cases with a documented tumor size, the mean size was $5.4 \mathrm{~cm}$ and the median size was $4 \mathrm{~cm}$ (Table 1). There were 92 tumors measuring $\leq 2 \mathrm{~cm}, 167$ tumors measuring $>2$ but $\leq 5 \mathrm{~cm}, 91$ tumors measuring $>5 \mathrm{~cm}$ but $\leq 10$ $\mathrm{cm}$, and 52 tumors measuring $>10 \mathrm{~cm}$. The largest tumor, which was in the colon, measured $29 \mathrm{~cm}$. Tumors in the colon/rectum were, on average, larger than tumors at other sites, with a mean tumor size of $7.8 \mathrm{~cm}$ in the colorectum versus $4.4 \mathrm{~cm}$ in the esophagus, $5.7 \mathrm{~cm}$ in the stomach, and $5.8 \mathrm{~cm}$ in the small bowel.

\section{Histologic features}

Most tumors exhibited no or mild cytologic atypia (281/407 cases, $69 \%$ ), while moderate atypia was seen in $56 / 407$ cases $(14 \%)$ and severe atypia was present in $70 / 407$ cases $(17 \%)$ (Table 1). Tumors were classified as closely resembling normal smooth muscle tissue (normal differentiation) in the majority of cases $(287 / 407,71 \%)$, but 78 cases $(19 \%)$ showed abnormal but identifiable smooth muscle differentiation and 42 cases $(10 \%)$ were classified as undifferentiated. Most cases demonstrated low cellularity (264/407 cases, $65 \%$ ), no tumor necrosis (309/407 cases, 76\%), and no mucosal ulceration (324/392 cases, 83\%). Calcifications, hyalinization, a prominent eosinophilic infiltrate, and/or an epithelioid component were also noted in subsets of cases.

Mitotic activity ranged from 0 to 310 mitoses $/ 5 \mathrm{~mm}^{2}$, with a mean of 13.9 mitoses $/ 5 \mathrm{~mm}^{2}$ and a median of 0 mitoses $/ 5 \mathrm{~mm}^{2}$. Lamina propria involvement was seen in $53 / 392$ cases $(14 \%)$ and serosal involvement was evident in $45 / 397$ cases (11\%) (Table 1). A positive margin was present in only $41 / 339$ cases (12\%).

\section{Clinical outcome}

The mean follow-up time for all cases was 64.4 months, with a median of 52 months (range 2-284 months). Progressive disease was detected in 56 patients (14\%), of whom half (28/56) died of disease. Progression was seen as early as 1 month after surgical resection and as late as 132 months following surgery; the average time to progression was 25.5 months. Of the 56 patients with progressive disease, nine had local recurrence alone, 35 had metastatic disease alone, four had both local recurrence and metastatic disease, and eight died of disease with an unknown intervening clinical course. The most common site of metastatic disease was the liver ( 21 cases), followed by the lung (11 cases) and peritoneum (10 cases). Rarer sites of involvement included bone, pancreas, bladder, brain, heart, mediastinum, spleen, and pelvis.

\section{Features associated with disease progression}

The following pathologic features were significantly associated with progressive disease on univariate analysis: tumor site, tumor size, mitotic count, moderate-to-severe atypia (sensitivity $91 \%$, specificity $79 \%$ ), high cellularity (sensitivity $96 \%$, specificity $75 \%$ ), abnormal differentiation or lack of differentiation (sensitivity $91 \%$, specificity $80 \%$ ), tumor necrosis (sensitivity $71 \%$, specificity $83 \%$ ), mucosal ulceration (sensitivity $37 \%$, specificity $86 \%$ ), lamina propria involvement (sensitivity $40 \%$, specificity $91 \%$ ), and serosal involvement (sensitivity $45 \%$, specificity $94 \%)(P<0.0001$ for all features) (Table 1). None of the patients with esophageal smooth muscle tumors experienced disease progression, while $24 / 56$ patients $(43 \%)$ with colorectal tumors had progressive disease; gastric and small bowel tumors showed intermediate numbers of progressive cases (14/180 [8\%] and 18/74 [24\%] cases, respectively). Age, sex, and margin status were not significantly associated with disease progression ( $P=0.23,0.82$, and 0.07 , respectively).

Receiver operator characteristic (ROC) analysis of continuous variables was used to identify optimal cutoffs to discriminate between progressive and non-progressive tumors. This analysis revealed an optimal cutoff of $9.8 \mathrm{~cm}$ for tumor size, which had a sensitivity of 0.63 and a specificity of 0.92 (Fig. 3). The ROC curve for mitotic activity revealed an optimal cutoff of $3 / 5 \mathrm{~mm}^{2}$, which had a sensitivity of 0.95 and a specificity of 0.86 (Fig. 4).

\section{Risk of progression of subgroups based on site, size, and mitotic activity}

Cases were divided into subgroups based on tumor site and the cutoff points identified by ROC analysis (tumor size cutoff of $10 \mathrm{~cm}$, mitotic activity cutoff of 3 mitoses $/ 5 \mathrm{~mm}^{2}$ ).

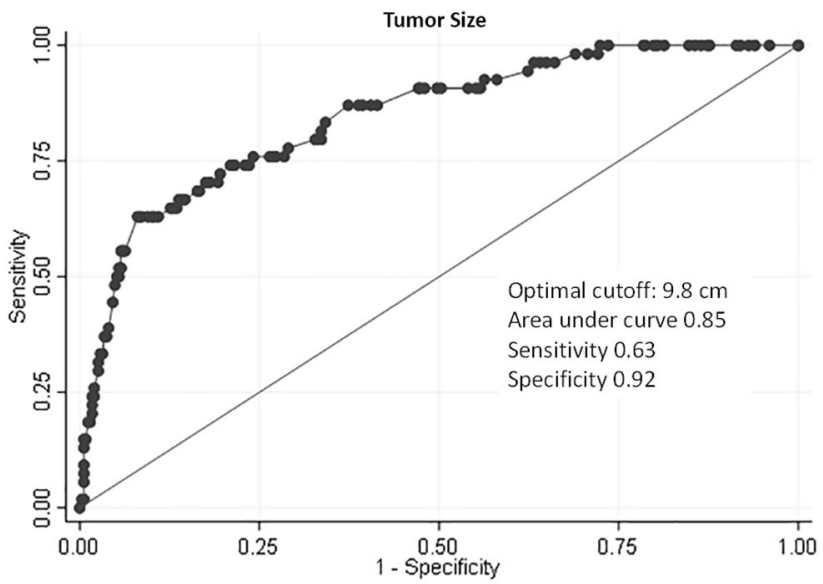

Fig. 3 Receiver operator characteristic (ROC) curve of tumor size to distinguish between progressive and non-progressive gastrointestinal smooth muscle tumors. 


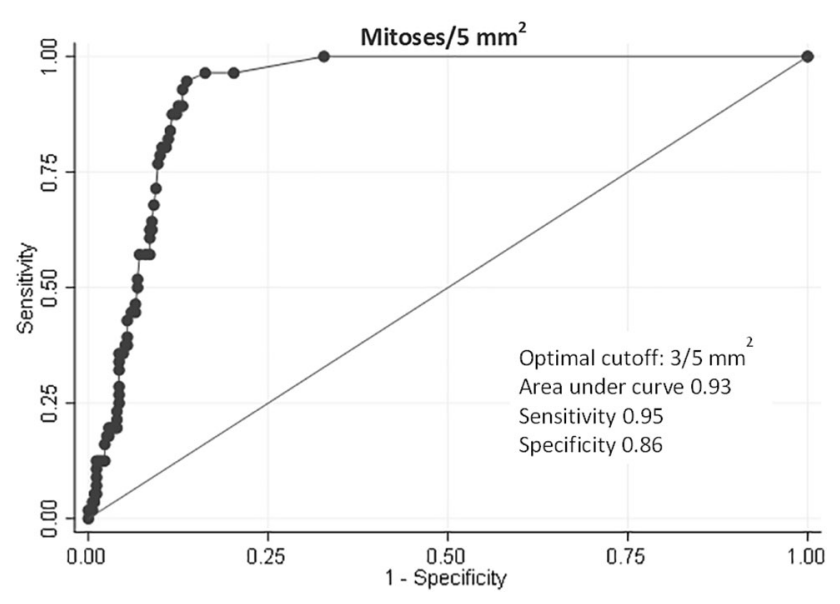

Fig. 4 Receiver operator characteristic (ROC) curve of mitoses $/ 5 \mathrm{~mm}^{2}$ to distinguish between progressive and non-progressive gastrointestinal smooth muscle tumors.

The Kaplan-Meier method was used to plot progression-free survival for each subgroup (Fig. 5). These plots revealed three progression-based tiers. The highest tier showed no disease progression, and included all esophageal smooth muscle tumors as well as tumors from all sites measuring $\leq 10 \mathrm{~cm}$ with $<3$ mitoses $/ 5 \mathrm{~mm}^{2}$. The three colorectal tumors that were $>10 \mathrm{~cm}$ but had $<3$ mitoses $/ 5 \mathrm{~mm}^{2}$ also fell into this top tier. The middle tier had early progression in a subset of cases $(<50 \%)$, with no progression seen in the remaining cases. This tier included large $(>10 \mathrm{~cm})$ gastric and small bowel tumors with $<3$ mitoses $/ 5 \mathrm{~mm}^{2}$ and small and medium $(\leq 10 \mathrm{~cm})$ gastric and small bowel tumors with $\geq 3$ mitoses $/ 5 \mathrm{~mm}^{2}$. The lowest tier showed progressive disease in $>50 \%$ of cases, with a steady increase in the number of progressive cases over time. This prognostically poor tier was composed of colorectal tumors of any size with a mitotic count of $\geq 3$ mitoses $/ 5 \mathrm{~mm}^{2}$, as well as large $(>10 \mathrm{~cm})$ gastric and small bowel tumors with $\geq 3$ mitoses $/ 5 \mathrm{~mm}^{2}$. The results of this analysis were also used to create a risk assessment table showing the risk of progressive disease for each subgroup, similar to that used for GISTs (Table 2).

Notably, none of the 92 smooth muscle tumors measuring $\leq 2 \mathrm{~cm}$ demonstrated disease progression, including 4 cases with $\geq 3$ mitoses $/ 5 \mathrm{~mm}^{2}$. The three smallest tumors to show progression were all located in the colorectum, with sizes of $2.2,2.5$, and $2.9 \mathrm{~cm}$. The smallest small bowel smooth muscle tumor to progress measured $3.0 \mathrm{~cm}$, and the smallest gastric smooth muscle tumor with progression measured $4.0 \mathrm{~cm}$.

\section{Discussion}

This study of 407 intramural GI smooth muscle tumors, the largest to date, examines the natural history of these neoplasms and identifies clinicopathologic features associated with their risk of progression. Previous studies of these tumors have been limited by small size and thus a lack of ability to generate meaningful prognostic data. By collecting cases from 31 institutions, we amassed a large cohort of GI smooth muscle tumors, allowing for more robust statistical analysis.

Based on our cohort, GI smooth muscle tumors appear to occur over a wide age range, though these tumors are most commonly encountered in middle-aged patients. While prior studies have suggested that smooth muscle tumors, particularly those located in the esophagus, are more common in males [6, 11, 12], our series revealed a slight female predominance across all sites. Similarly, the stomach was the most common location of tumors in our study, which was surprising given that esophageal smooth muscle tumors are widely considered most common and prior reports have indicated that gastric smooth muscle tumors are quite rare $[9,11,13]$. The reason for this discrepancy is not entirely clear, but it is possible that the exclusion of tumors measuring less than $0.5 \mathrm{~cm}$ contributed to the small number of esophageal tumors in our study, as prior studies have indicated that minute tumors are quite common at this site [15]. In addition, the requirement for immunohistochemical confirmation of smooth muscle differentiation may have resulted in inclusion of fewer esophageal tumors in our study, as immunohistochemical stains are less likely to have been performed in tumors from this site. Based on our findings, however, intramural smooth muscle tumors of the stomach do not appear to be as uncommon as previously thought; immunohistochemical confirmation of tumor cell differentiation in tumors with high-risk features is therefore important, as the therapeutic agents used to treat malignant smooth muscle tumors and GISTs differ greatly. Of note in this regard, desmin has been shown to be a more specific marker for smooth muscle tumors than smooth muscle actin, as GISTs can occasionally demonstrate smooth muscle actin positivity $[11,16]$. In addition, smooth muscle tumors of the GI tract can be colonized by interstitial cells of Cajal, resulting in scattered cells with KIT staining, which should not be misinterpreted as diagnostic of a GIST [13].

The presence of multifocal GI tract smooth muscle tumors was uncommon in our study, and multifocality was not seen in any patients with known genetic tumorpredisposing conditions. Three patients with unifocal tumors had MEN1, which is known to be associated with the development of smooth muscle tumors in multiple organs, including the esophagus $[13,17]$; interestingly, two of the MEN1 patients in our study had small bowel smooth muscle tumors, which is not a site of involvement previously reported in the literature. Three other patients in the study had Lynch syndrome, which is not known to be 


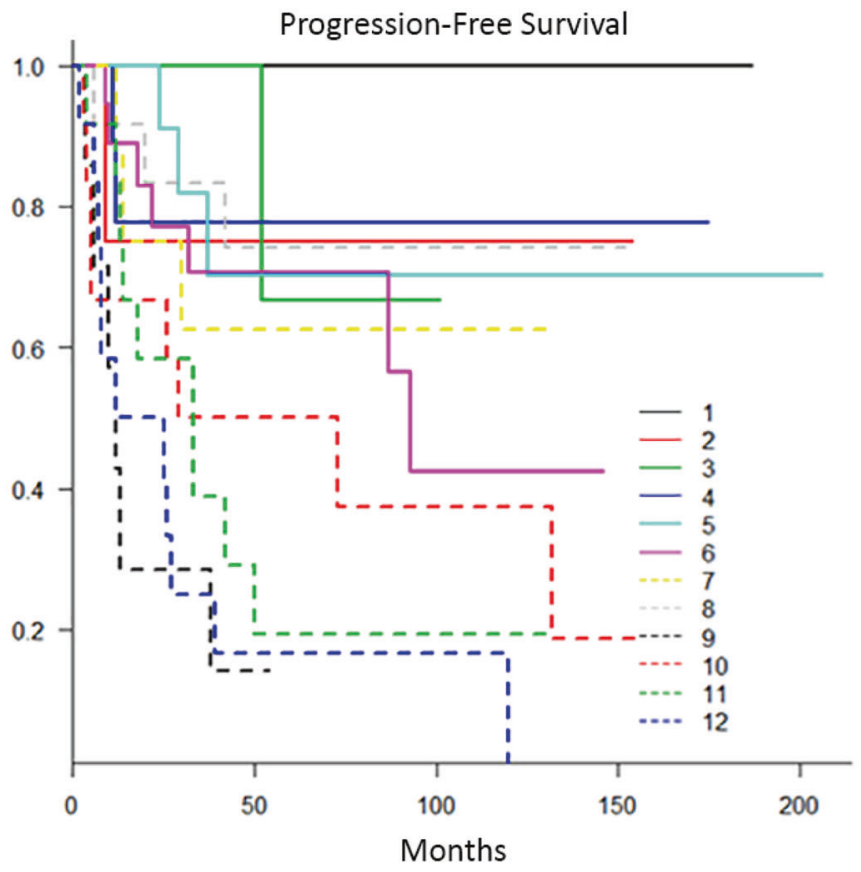

\begin{tabular}{|l|l|l|l|l|}
\cline { 2 - 5 } \multicolumn{1}{l|}{} & Site & Size & Mitotic Rate & $\%$ Progression \\
\hline- & All & $\leq 10 \mathrm{~cm}$ & $<3 / 5 \mathrm{~mm}^{2}$ & $0 \%(0 / 280)$ \\
\hline- & Esophagus & $>10 \mathrm{~cm}$ & $<3 / 5 \mathrm{~mm}^{2}$ & $0 \%(0 / 5)$ \\
\hline- & Stomach & $>10 \mathrm{~cm}$ & $<3 / 5 \mathrm{~mm}^{2}$ & $25 \%(1 / 4)$ \\
\hline- & Small bowel & $>10 \mathrm{~cm}$ & $<3 / 5 \mathrm{~mm}^{2}$ & $33 \%(1 / 3)$ \\
\hline- & Colon/Rectum & $>10 \mathrm{~cm}$ & $<3 / 5 \mathrm{~mm}^{2}$ & $0 \%(0 / 3)$ \\
\hline- & Esophagus & $\leq 5 \mathrm{~cm}$ & $23 / 5 \mathrm{~mm}^{2}$ & $0 \%(0 / 3)$ \\
\hline- & Stomach & $\leq 5 \mathrm{~cm}$ & $\geq 3 / 5 \mathrm{~mm}^{2}$ & $22 \%(2 / 9)$ \\
\hline- & Small bowel & $\leq 5 \mathrm{~cm}$ & $\geq 3 / 5 \mathrm{~mm}^{2}$ & $23 \%(3 / 13)$ \\
\hline- & Colon/Rectum & $\leq 5 \mathrm{~cm}$ & $\geq 3 / 5 \mathrm{~mm}^{2}$ & $39 \%(7 / 18)$ \\
\hline & Esophagus & $>5 \& \leq 10 \mathrm{~cm}$ & $23 / 5 \mathrm{~mm}^{2}$ & No cases \\
\hline--- & Stomach & $>5 \& \leq 10 \mathrm{~cm}$ & $\geq 3 / 5 \mathrm{~mm}^{2}$ & $38 \%(3 / 8)$ \\
\hline--- & Small bowel & $>5 \& \leq 10 \mathrm{~cm}$ & $23 / 5 \mathrm{~mm}^{2}$ & $25 \%(3 / 12)$ \\
\hline--- & Colon/Rectum & $>5 \& \leq 10 \mathrm{~cm}$ & $\geq 3 / 5 \mathrm{~mm}^{2}$ & $86 \%(6 / 7)$ \\
\hline- & Esophagus & $>10 \mathrm{~cm}$ & $23 / 5 \mathrm{~mm}^{2}$ & $0 \%(0 / 1)$ \\
\hline--- & Stomach & $>10 \mathrm{~cm}$ & $23 / 5 \mathrm{~mm}^{2}$ & $67 \%(8 / 12)$ \\
\hline--- & Small bowel & $>10 \mathrm{~cm}$ & $\geq 3 / 5 \mathrm{~mm}^{2}$ & $75 \%(9 / 12)$ \\
\hline--- & Colon/Rectum & $>10 \mathrm{~cm}$ & $\geq 3 / 5 \mathrm{~mm}^{2}$ & $92 \%(11 / 12)$ \\
\hline
\end{tabular}

Fig. 5 Kaplan-Meier plot of progression-free survival for subgroups of gastrointestinal smooth muscle tumors

Table 2 Risk assessment of gastrointestinal smooth muscle tumors.

\begin{tabular}{|c|c|c|c|c|c|}
\hline \multicolumn{2}{|c|}{ Tumor parameters } & \multicolumn{4}{|c|}{ Risk of progressive disease } \\
\hline Mitotic rate & Size & Esophagus & Stomach & Small bowel & Colorectum \\
\hline \multirow[t]{3}{*}{$<3$ per $5 \mathrm{~mm}^{2}$} & $\leq 5 \mathrm{~cm}$ & $0 \%(0 / 62)$ & $0 \%(0 / 117)$ & $0 \%(0 / 24)$ & $0 \%(0 / 13)$ \\
\hline & $>5$ and $\leq 10 \mathrm{~cm}$ & $0 \%(0 / 26)$ & $0 \%(0 / 29)$ & $0 \%(0 / 7)$ & $0 \%(0 / 2)$ \\
\hline & $>10 \mathrm{~cm}$ & $0 \%(0 / 5)$ & $25 \%(1 / 4)$ & $33 \%(1 / 3)$ & $0 \%(0 / 3)$ \\
\hline \multirow[t]{3}{*}{$\geq 3$ per $5 \mathrm{~mm}^{2}$} & $\leq 5 \mathrm{~cm}$ & $0 \%(0 / 3)$ & $22 \%(2 / 9)$ & $23 \%(3 / 13)$ & $39 \%(7 / 18)$ \\
\hline & $>5$ and $\leq 10 \mathrm{~cm}$ & No cases & $38 \%(3 / 8)$ & $25 \%(3 / 12)$ & $86 \%(6 / 7)$ \\
\hline & $>10 \mathrm{~cm}$ & $0 \%(0 / 1)$ & $67 \%(8 / 12)$ & $75 \%(9 / 12)$ & $92 \%(11 / 12)$ \\
\hline
\end{tabular}

associated with the development of smooth muscle tumors. However, given the frequency of GI screening and surgery in patients with this syndrome, asymptomatic smooth muscle tumors may be detected at a higher rate in patients with Lynch syndrome than in the general population.

Despite these differences, our study does support the impressions from prior smaller studies and anecdotal data that the majority of intramural smooth muscle tumors of the GI tract are biologically benign, though aggressively behaving tumors do occur, and the risk of such behavior is largely site-dependent. Progression of esophageal smooth muscle tumors is extremely rare, with none of the 97 esophageal tumors in this study, which ranged in size from 0.5 to $15 \mathrm{~cm}$ and mostly (93/97) had $<3$ mitoses $/ 5 \mathrm{~mm}^{2}$, demonstrating disease progression. However, aggressive esophageal smooth muscle tumors, most of which were large and mitotically active, have been described in the literature, so these lesions cannot be deemed universally benign [6]. Colorectal smooth muscle tumors, in contrast, showed a much higher proclivity for aggressive behavior, with disease progression seen in $43 \%$ of cases in this study; these tumors ranged in size from 2.2 to $29 \mathrm{~cm}$, and all had $\geq 3$ mitoses $/ 5 \mathrm{~mm}^{2}$. Small bowel and gastric tumors demonstrated intermediate rates of progression.

Several histologic features were strongly associated with aggressive behavior in GI smooth muscle tumors, including moderate-to-severe atypia, high cellularity, abnormal differentiation or lack of differentiation, tumor necrosis, mucosal ulceration, lamina propria involvement, and serosal involvement. Notably, moderate-to-severe atypia, high cellularity, and abnormal differentiation/lack of differentiation were each present in more than $90 \%$ of cases with disease progression, and only one tumor exhibiting mild atypia, low cellularity, and normal differentiation demonstrated disease progression; this colorectal tumor measured $20 \mathrm{~cm}$, had 6 mitoses $/ 5 \mathrm{~mm}^{2}$, and recurred locally. It is therefore safe to assume that small GI smooth muscle tumors without significant atypia, hypercellularity, abnormal differentiation, or 
an elevated mitotic rate will not behave aggressively. While tumor necrosis, mucosal ulceration, lamina propria involvement, and serosal involvement were also significantly associated with disease progression, these features were seen in smaller percentages of progressive cases and appear to be less sensitive markers of aggressive behavior. In addition, although the association between margin status and disease progression did not reach statistical significance $(P=0.07)$, the small number of cases with a positive margin (41/335) may have limited our ability to detect a statistically significant difference between groups. While histologic assessment of margin status is often difficult in these cases, it is reasonable to assume that patients with a truly positive margin have an increased risk of local recurrence, especially in cases with other high-risk features.

As expected, tumor size and mitotic activity were also significantly associated with disease progression of GI smooth muscle tumors, and ROC analysis revealed a rounded tumor size of $10 \mathrm{~cm}$ and a mitotic count of $3 / 5 \mathrm{~mm}^{2}$ to be optimal cutoffs for predicting aggressive behavior in these tumors. Kaplan-Meier plots of subgroups based on these cutoffs and tumor site roughly divided the tumors into high-, intermediate-, and low-risk groups (Fig. 5).

Our analysis, therefore, highlights the presence of distinct subgroups of GI smooth muscle tumors, with indolent and aggressive counterparts that can be identified based on tumor site, size, and mitotic rate (Table 2). However, as is the case for GISTs, there appears to be a spectrum to the biologic behavior of these tumors, with an intermediate-risk group for which the clinical course is more difficult to predict. Firm categorization of cases as benign and malignant is thus not always feasible, and instead assessment of progression risk using the table provided is encouraged. Based on our findings, it is certainly reasonable to label GI tract smooth muscle tumors smaller than $10 \mathrm{~cm}$ with $<3$ mitoses $/ 5 \mathrm{~mm}^{2}$ as "leiomyomas" and GI tract smooth muscle tumors larger than $10 \mathrm{~cm}$ with $\geq 3$ mitoses $/ 5 \mathrm{~mm}^{2}$ as "leiomyosarcomas." However, application of this nomenclature requires use of a third term to describe tumors in the intermediate-risk group- "atypical smooth muscle tumor" has previously been proposed and may be most appropriate. In the interest of consistency with the terminology used for GISTs, some pathologists may prefer to use the term "smooth muscle tumor" for all intramural smooth muscle neoplasms of the GI tract. Regardless of the terminology used, inclusion of a description of the histologic features of the lesion (including size, mitotic rate, atypia, cellularity, differentiation, necrosis, ulceration, lamina propria invasion, and serosal invasion) in the pathology report is recommended, since such features appear to play a role in the graded biologic behavior of these tumors.

Further analysis of the subset of tumors with intermediate risk of progression is also needed, and molecular analysis may prove a particularly useful tool in helping to predict the behavior of these tumors. Indeed, a recent study of uterine smooth muscle tumors of uncertain malignant potential found that grouping such tumors according to a genomic index based on array comparative genomic hybridization results correlated well with patient outcome [18]. Next-generation sequencing of GI smooth muscle tumors as a whole may also allow for identification of specific alterations associated with aggressive behavior.

Several limitations of this study must be noted. There was no central review of the cases, though electronic images were distributed and a robust online discussion was held prior to case review and data collection to minimize discrepancies in evaluation of histologic features. In addition, due to the infrequent use of immunohistochemical stains in the diagnosis of esophageal smooth muscle tumors and the frequency of minute tumors at this location, many intramural esophageal smooth muscle tumors were probably excluded from our study, and the proportion of these tumors may therefore be artificially low. More importantly, while the "intramural" smooth muscle tumors included in this study were presumed to arise from the muscularis propria, and cases that clearly arose from mesenteric vessels were excluded, it is possible that some of the tumors arose from a vessel wall within the muscle layer. Whether such tumors should be considered "GI" smooth muscle tumors and whether these tumors should be evaluated using the same criteria as tumors arising from the muscle itself are both debatable questions. In addition, while cases with fewer than 12 months of clinical follow-up and no progression were excluded from the study, given that the average time to progression was 25.5 months and progression in 31/56 (55\%) progressive cases occurred after 12 months, this minimum follow-up time was likely insufficient to detect all progressive cases. Finally, despite our efforts to amass a large number of GI smooth muscle tumors, the number of progressive cases collected was insufficient to perform multivariate analysis.

Despite these limitations, the results of this analysis of 407 intramural GI smooth muscle tumors enhances our understanding of the natural history of these neoplasms, and also improves practicing pathologists' ability to identify tumors at risk of progression based on their histologic features. From a clinicopathologic standpoint, our data indicate that nonesophageal tumors measuring $>10 \mathrm{~cm}$ and/ or showing $\geq 3$ mitoses $/ 5 \mathrm{~mm}^{2}$ may behave aggressively, and therefore close follow-up can be recommended in these cases. Colorectal smooth muscle tumors in general have the highest risk of progression, followed by small bowel and gastric tumors, while esophageal tumors are unlikely to demonstrate aggressive behavior. Future studies, including those involving molecular analysis, may allow for additional stratification of intermediate-risk tumors. 


\section{Compliance with ethical standards}

Conflict of interest The authors declare that they have no conflict of interest.

Publisher's note Springer Nature remains neutral with regard to jurisdictional claims in published maps and institutional affiliations.

\section{References}

1. Miettinen M, Lasota J. Gastrointestinal stromal tumors: pathology and prognosis at different sites. Semin Diagn Pathol. 2006;23: 70-83.

2. Miettinen M, Sobin LH, Lasota J. Gastrointestinal stromal tumors of the stomach: a clinicopathologic, immunohistochemical, and molecular genetic study of 1765 cases with long-term follow-up. Am J Surg Pathol. 2005;29:52-68.

3. Miettinen M, Makhlouf H, Sobin LH, Lasota J. Gastrointestinal stromal tumors of the jejunum and ileum: a clinicopathologic, immunohistochemical, and molecular genetic study of 906 cases before imatinib with long-term follow-up. Am J Surg Pathol. 2006;30:477-89.

4. College of American Pathologists. Protocol for the examination of resection specimens for patients with gastrointestinal stromal tumor (GIST). Version 4.1.0.0. Northfield (IL): College of American Pathologists; 2019. https://documents.cap.org/protocols/cp-othergist-resection-19-4100.pdf.

5. Miettinen M, Sarlomo-Rikala M, Sobin LH, Lasota J. Gastrointestinal stromal tumors and leiomyosarcomas in the colon: a clinicopathologic, immunohistochemical, and molecular genetic study of 44 cases. Am J Surg Pathol. 2000;24:1339-52.

6. Miettinen M, Sarlomo-Rikala M, Sobin LH, Lasota J. Esophageal stromal tumors: a clinicopathologic, immunohistochemical, and molecular genetic study of 17 cases and comparison with esophageal leiomyomas and leiomyosarcomas. Am J Surg Pathol. 2000;24:211-22.

7. Miettinen M, Kopczynski J, Makhlouf HR, Sarlomo-Rikala M, Gyorffy H, Burke A, et al. Gastrointestinal stromal tumors, intramural leiomyomas, and leiomyosarcomas in the duodenum: a clinicopathologic, immunohistochemical, and molecular genetic study of 167 cases. Am J Surg Pathol. 2003;27:625-41.

8. Miettinen M, Fetsch JF. Evaluation of biological potential of smooth muscle tumours. Histopathology. 2006;48:97-105.

9. Agaimy A, Wünsch PH. True smooth muscle neoplasms of the gastrointestinal tract: morphological spectrum and classification in a series of 85 cases from a single institute. Langenbecks Arch Surg. 2007;392:75-81.

10. Miettinen M, Sobin LH, Lasota J. True smooth muscle tumors of the small intestine: a clinicopathologic, immunhistochemical, and molecular genetic study of 25 cases. Am J Surg Pathol. 2009; 33:430-6.

11. Aggarwal G, Sharma S, Zheng M, Reid MD, Crosby JH, Chamberlain SM, et al. Primary leiomyosarcomas of the gastrointestinal tract in the post-gastrointestinal stromal tumor era. Ann Diagn Pathol. 2012;16:532-40.

12. Yamamoto H, Handa M, Tobo T, Setsu N, Fujita K, Oshiro Y, et al. Clinicopathological features of primary leiomyosarcoma of the gastrointestinal tract following recognition of gastrointestinal stromal tumours. Histopathology. 2013;63:194-207.

13. Miettinen M. Smooth muscle tumors of soft tissue and non-uterine viscera: biology and prognosis. Mod Pathol. 2014;27:S17-29.

14. Trojani M, Contesso G, Coindre JM, Rouesse J, Bui NB, de Mascarel A, et al. Soft-tissue sarcomas of adults; study of pathological prognostic variables and definition of a histopathological grading system. Int J Cancer. 1984;33:37-42.

15. Abraham SC, Krasinskas AM, Hofstetter WL, Swisher SG, Wu TT. "Seedling" mesenchymal tumors (gastrointestinal stromal tumors and leiomyomas) are common incidental tumors of the esophagogastric junction. Am J Surg Pathol. 2007;31:1629-35.

16. Weiss SW. Smooth muscle tumors of soft tissue. Adv Anat Pathol. 2002;9:351-9.

17. McKeeby JL, Li X, Zhuang $Z$, Vortmeyer AO, Huang S, Pirner M, et al. Multiple leiomyomas of the esophagus, lung, and uterus in multiple endocrine neoplasia type 1. Am J Pathol. 2001; 159:1121-7.

18. Croce S, Ducoulombier A, Ribeiro A, Lesluyes T, Noel JC, Amant F, et al. Genome profiling is an efficient tool to avoid the STUMP classification of uterine smooth muscle lesions: a comprehensive array-genomic hybridization analysis of 77 tumors. Mod Pathol. 2018;31:816-28.

\section{Affiliations}

Lindsay Alpert ${ }^{1} \cdot$ Ram Al-Sabti ${ }^{1} \cdot$ Rondell P. Graham $\mathbb{D}^{2} \cdot$ Rish K. Pai $\mathbb{D}^{3} \cdot$ Raul S. Gonzalez $\mathbb{C}^{4} \cdot$ Xuefeng Zhang $^{5}$. Vanessa Smith $\mathbb{D}^{5} \cdot$ Hanlin L. Wang ${ }^{6} \cdot$ Lindsey Westbrook $\mathbb{D}^{6} \cdot$ John R. Goldblum ${ }^{7} \cdot$ Ahmed Bakhshwin ${ }^{7}$. Sindhu Shetty ${ }^{7}$ - David S. Klimstra ${ }^{8}$. Jinru Shia $\square^{8} \cdot$ Gokce Askan $^{8}$ - Marie E. Robert ${ }^{9}$ - Courtney Thomas $^{9}$. Wendy L. Frankel ${ }^{10} \cdot$ Mohammed Alsomali $^{10}$ - Catherine Hagen ${ }^{11} \cdot$ Mohamed E. Mostafa $^{11}$ - Michael M. Feely ${ }^{12}$. Naziheh Assarzadegan ${ }^{12} \cdot$ Joseph Misdraji $^{13}$ - Angela R. Shih ${ }^{13}$. Diana Agostini-Vulaj $\mathbb{D}^{14} \cdot$ Jeanne M. Meis ${ }^{15}$. Sherry Tang ${ }^{15}$. Deyali Chatterjee ${ }^{16}$. Liang-I Kang ${ }^{16}$. John Hart ${ }^{1}$. Sang Mee Lee ${ }^{1}$. Theresa Smith ${ }^{17}$. Rhonda K. Yantiss ${ }^{18}$ • Erika M. Hissong ${ }^{18} \cdot$ Zu-hua Gao $^{19}$ - JingBo Wu ${ }^{19}$. Murray B. Resnick ${ }^{20}$. Elizabeth Yiru Wu ${ }^{20}$. Reet K. Pai ${ }^{21}$ - Lei Zhao ${ }^{22}$ - Leona A. Doyle ${ }^{22}$. Shefali Chopra ${ }^{23}$ - Nicole C. Panarelli ${ }^{24}$. Shaomin $\mathrm{Hu}^{24}$. Teri A. Longacre ${ }^{25}$. Shyam Sampath Raghavan $\mathbb{D}^{25} \cdot$ Gregory Y. Lauwers $^{26}$ - Masoumeh Ghayouri ${ }^{26}$. Harry S. Cooper ${ }^{27}$ - Rajeswari Nagarathinam ${ }^{27}$ - Andrew M. Bellizzi ${ }^{28}$. Sanjay Kakar ${ }^{29} \cdot$ Mojgan Hosseini $^{30}$. Juan Rong ${ }^{30} \cdot$ Joel K. Greenson ${ }^{31} \cdot$ Laura W. Lamps $^{31} \cdot$ Zachary Dong $^{32} \cdot$ Mary P. Bronner $^{32}$

1 University of Chicago, Chicago, IL, USA

2 Mayo Clinic, Rochester, MN, USA

3 Mayo Clinic, Scottsdale, AZ, USA
4 Beth Israel Deaconess Medical Center, Boston, MA, USA

Duke University Medical Center, Durham, NC, USA 
6 UCLA David Geffen School of Medicine, Los Angeles, CA, USA

7 Cleveland Clinic Foundation, Cleveland, OH, USA

8 Memorial Sloan Kettering Cancer Center, New York, NY, USA

9 Yale University School of Medicine, New Haven, CT, USA

10 The Ohio State University Wexner Medical Center, Columbus, OH, USA

11 Medical College of Wisconsin, Milwaukee, WI, USA

12 University of Florida, Gainesville, FL, USA

13 Massachusetts General Hospital, Boston, MA, USA

14 University of Rochester Medical Center, Rochester, NY, USA

15 The University of Texas MD Anderson Cancer Center, Houston, TX, USA

16 Washington University School of Medicine, St. Louis, MO, USA

17 Roswell Park Comprehensive Cancer Center, Buffalo, NY, USA

18 Weill Cornell Medicine, New York, NY, USA

19 McGill University, Montreal, Quebec, Canada
20 Lifespan/The Warren Alpert Medical School of Brown University, Providence, RI, USA

21 University of Pittsburgh Medical Center, Pittsburgh, PA, USA

22 Brigham and Women's Hospital, Boston, MA, USA

23 Keck Medical Center, University of Southern California, Los Angeles, CA, USA

24 Montefiore Medical Center, Bronx, NY, USA

25 Stanford University, Stanford, CA, USA

26 Moffitt Cancer Center, Tampa, FL, USA

27 Fox Chase Cancer Center, Philadelphia, PA, USA

28 University of Iowa Health Care, Iowa City, IA, USA

29 University of California, San Francisco, San Francisco, CA, USA

30 University of California, San Diego, La Jolla, CA, USA

31 University of Michigan, Ann Arbor, MI, USA

32 University of Utah, Salt Lake City, UT, USA 